\title{
Experiences of a One-hour Algorithm in Chest Pain Patients With a Nonelevated Troponin T at Presentation
}

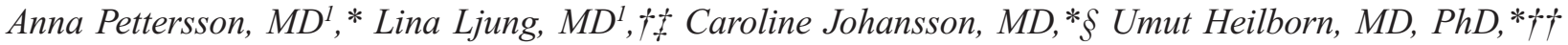 \\ Tomas Jernberg, MD, PhD, \ Mats Frick, MD, PhD, †ł Kai M. Eggers, MD, PhD, \| Bertil Lindahl, MD, PhD, $\|^{* * *}$

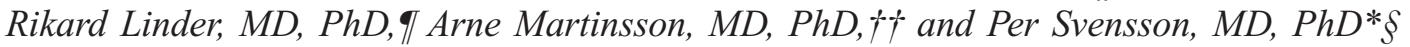

\begin{abstract}
Background: We aimed to evaluate the use of a 1-hour measurement of high-sensitivity cardiac troponin $\mathrm{T}$ (hs-cTnT) in an emergency department (ED) population of chest pain patients with a nonelevated baseline hs-cTnT and to examine the prevalence of early dynamic changes in hs-cTnT and the association with admission rate, diagnosis, and outcome.

Methods: All patients with a chief complaint of chest pain presenting to the ED of Karolinska University Hospital, Solna, Sweden, from December 2014 to September 2015 who had a baseline hs-cTnT of $\leq 14 \mathrm{ng} / \mathrm{L}$ and a second value obtained within $>30$ to $\leq 90$ minutes were followed for 30 days regarding admission, readmission, myocardial infarction (MI), and death.

Results: A total of 1091 patients were included. Dynamic 1-hour changes in hs-cTnT defined as an increase or decrease of $\geq 3 \mathrm{ng} / \mathrm{L}$ occurred in 23 patients $(2.1 \%)$. Fifteen patients $(65.2 \%)$ in the dynamic group were admitted, compared with 148 patients $(13.9 \%)$ in the nondynamic group $(P$ $<0.001)$. Four of the admitted patients $(26.7 \%)$ in the dynamic and $1(0.7 \%)$ in the nondynamic group were diagnosed with an MI $(P<0.001)$. No death or MI occurred within 30 days among those discharged from the ED.

Conclusions: Dynamic 1-hour changes in hs-cTnT were uncommon but associated with a higher rate of admission and of $\mathrm{MI}$ in an unselected population of chest pain patients with a nonelevated hs-cTnT at presentation. Lack of dynamic changes makes MI highly unlikely, and a 1-hour measurement may facilitate an early rule out of MI but should be used together with clinical assessment.
\end{abstract}

Key Words: 1-hour algorithm, chest pain, emergency department, myocardial infarction, troponin

(Crit Pathways in Cardiol 2018;17: 6-12)

Received for publication June 4, 2017; accepted September 23, 2017

From the *Department of Medicine, Solna, Karolinska Institutet, Sweden; $\uparrow$ Department of Clinical Science and Education, Södersjukhuset, Karolinska Institutet, Sweden; ‡Department of Cardiology, Södersjukhuset, Sweden; §Functional Area of Emergency Medicine, Karolinska University Hospital Solna, Sweden; IDepartment of Clinical Sciences, Danderyd University Hospital, Karolinska Institutet, Sweden; \|Department of Medical Sciences, Uppsala University, Sweden; **Uppsala Clinical Research Center, Uppsala University, Sweden; and $\dagger$ †Department of Emergency Medicine, Capio S:t Göran's Hospital, Sweden.

'Anna Pettersson and Lina Ljung share first authorship.

Kai M. Eggers has received honoraria from Abbott Laboratories and AstraZeneca and has served as a consultant for Abbott Laboratories and Fiomi Diagnostics. Bertil Lindahl has received consulting fees from Roche Diagnostics, bioMérieux Clinical Diagnostics, Philips Healthcare, ThermoFischer, and Fiomi Diagnostics and has received a research grant from bioMérieux Clinical Diagnostics and Fiomi Diagnostics. For the remaining authors, none were declared. This research was supported by grants provided by the Swedish Heart-Lung Foundation and the Stockholm County Council (ALF project).

Supplemental digital content is available for this article. Direct URL citations appear in the printed text and are provided in the HTML and PDF versions of this article on the journal's Web site (www.critpathcardio.com).

Reprints: Lina Ljung, MD, Department of Clinical Science and Education, Södersjukhuset, Karolinska Institutet, Department of Cardiology, Södersjukhuset, S-118 83 Stockholm, Sweden. E-mail: lina.ljung@sll.se.

Copyright (C) 2018 The Author(s). Published by Wolters Kluwer Health, Inc. This is an open-access article distributed under the terms of the Creative Commons Attribution-Non Commercial-No Derivatives License 4.0 (CCBY-NC-ND), where it is permissible to download and share the work provided it is properly cited. The work cannot be changed in any way or used commercially without permission from the journal.

ISSN: 1003-0117/18/1701-0006

DOI: $10.1097 / \mathrm{HPC} .0000000000000138$
C hest pain is a common symptom among patients presenting to the emergency department (ED). ${ }^{1}$ Although acute coronary syndrome (ACS) can be ruled out in a majority of these patients, a few will eventually be diagnosed with an ongoing ACS. ${ }^{2}$ Notably, approximately $1 \%$ of patients discharged from the ED with unspecified chest pain experience a major adverse cardiac event (MACE) within 30 days. $^{3}$ It is of great importance to find a reliable algorithm for early rule in and rule out of ACS in chest pain patients, enabling an early initiation of treatment for those with an ongoing ACS and an early discharge for those where ACS can be ruled out.

The high-sensitivity cardiac troponin (hs-cTn) assays have markedly improved the accuracy of the test and made early testing more reliable. ${ }^{4}$ While conventional troponin assays require sampling 6 to 12 hours after presentation to safely rule out an ongoing myocardial infarction (MI), 3 to 6 hours from symptom onset has been considered sufficient in the algorithms using hs-cTn. ${ }^{5,6}$ In 2012, a 1-hour hs-cTn algorithm in chest pain patients was suggested ${ }^{7}$ and later validated. ${ }^{2,8}$ The European Society of Cardiology has recently presented this algorithm as an alternative to traditional assessment. Still, there is a lack of studies investigating early dynamic changes in troponin in the clinical setting, in particular, in patients with a nonelevated troponin at presentation.

The aim of the present study was to evaluate the use of a 1-hour measurement in clinical routine in an unselected ED population of chest pain patients with a nonelevated high-sensitivity cardiac troponin $\mathrm{T}$ (hs-cTnT) at presentation. Further, we studied whether early dynamic changes in hs-cTnT were associated with admission rate, subsequent diagnosis, and outcome.

\section{METHODS}

A new algorithm for the assessment of chest pain patients in the ED was introduced in clinical routine at Karolinska University Hospital, Solna, Sweden, on December 1, 2014. The algorithm was derived from the algorithm presented by Reichlin et $\mathrm{al}^{7}$ in 2012 . In all patients presenting with symptoms suggestive of ACS, regardless if the suspicion of ACS was low, intermediate, or high, and for whom analysis of hs-cTnT was initiated, a second sample should routinely be obtained after 1 hour. The attending physician had the possibility to omit the second sample in case it was considered redundant but did not routinely assess the patient before the second sample. ${ }^{9}$ In patients with a baseline hs-cTnT within normal reference range (ie, $\leq 14 \mathrm{ng} / \mathrm{L}$ ), the absolute change in hs-cTnT ( $\Delta$ hs-cTnT) was calculated and categorized as nondynamic if $<3 \mathrm{ng} / \mathrm{L}$ and dynamic if $\geq 3 \mathrm{ng} / \mathrm{L}$. The attending physician was also recommended to riskstratify the patients using the History, Electrocardiogram (ECG), Age, Risk factors and initial Troponin (HEART) score. The HEART score is prospectively validated for risk stratification of chest pain patients in the ED and includes the variables history, ECG, age, risk factors, and troponin. ${ }^{10}$ A HEART score of $\leq 3$ points is associated with a very low acute risk of a MACE, whereas a score of $>3$ is associated with an elevated acute risk. ${ }^{10-12}$ In patients with a baseline level of hs-cTnT of $\leq 14 \mathrm{ng} / \mathrm{L}$ and a dynamic 1 -hour change in hs-cTnT, the algorithm suggested consideration of an ongoing MI and admission. In patients with 
a baseline level of hs-cTnT of $\leq 14 \mathrm{ng} / \mathrm{L}$ and a nondynamic change, the assessment depended on the HEART score. In patients with a HEART score of $>3$ points, admission should be considered and those with a HEART score of $\leq 3$ were recommended to be discharged after consideration of differential diagnoses.

The patient selection in this retrospective observational study was made through the Karolinska University Hospital Database (KARDA), which consists of data from the hospital's electronic medical records. All patients who were registered with a chief complaint of chest pain when presenting to the ED of Karolinska University Hospital, Solna, Sweden, from December 1, 2014, to September 14, 2015, were screened for inclusion. The following inclusion criteria were applied: ${ }^{1}$ registered chief complaint of chest pain categorized by a triage nurse, ${ }^{2}$ age $>18$ years, ${ }^{3}$ a Swedish identity number, ${ }^{4} 2$ hs-cTnT measurements obtained during the ED visit with a time period between the first and second sample of $>30$ minutes but $\leq 90$ minutes. The same time interval was used as in the first clinical study evaluating the original algorithm. ${ }^{8}$ Patients were excluded if they had ST-segment-elevation MI or ventricular tachycardia at presentation, and these patients routinely bypassed the ED and were admitted directly to the coronary care unit or catheterization laboratory. For patients with several ED visits during the study period, one unique visit was randomly chosen and included in the analyses. Data extracted from the KARDA included baseline characteristics, time of arrival and time of stay in the ED, time until the physician met the patient, the attending physician's interpretation of the ECG and the result, and time of blood samples drawn in the ED. The interpretation of the ECG was reviewed by the authors. The time of onset of chest pain was not available. Hs-cTnT was analyzed with a Roche-Modular E or a Roche-Cobas 8000 e602 from Roche Diagnostics (Basel, Switzerland). The assay has a limit of blank of $3 \mathrm{ng} / \mathrm{L}$ and a limit of detection of $5 \mathrm{ng} / \mathrm{L}$, a 99 th percentile cutoff point of $14 \mathrm{ng} / \mathrm{L}$, and a coefficient of variation of less than or at $10 \%$ at the upper reference limit. ${ }^{13}$ The renal function expressed as estimated glomerular filtration rate was measured with the modification of diet in renal disease formula. ${ }^{14}$ To characterize all patients with a dynamic change in hs-cTnT, as well as those diagnosed with ACS without a dynamic change, the HEART score was calculated retrospectively by the authors using the medical records and read-outs of admission ECGs. The authors were blinded to hs-cTnT and outcome to avoid bias.

All patients with a baseline hs-cTnT value of $\leq 14 \mathrm{ng} / \mathrm{L}$ were followed for 30 days regarding admission, readmission to the Karolinska University Hospital, Solna or Huddinge, and death. This information, including diagnoses coded according to the International Classification of Diseases, was extracted from the KARDA with a linkage to the Swedish population register. In all patients with a main discharge diagnosis of I21 or I22, the diagnosis and type (1 or 2) of MI was adjudicated by 2 independent cardiologists.

Continuous variables were presented as mean with standard deviation or median with interquartile range (IQR) or minimum and maximum range (min-max range) as appropriate, and comparisons were made with a Student $t$ test for normally distributed variables and with a Mann-Whitney $U$ test for other continuous variables. Categorical variables were given as numbers and percentages, and comparisons were made with Pearson's $\chi^{2}$ test or Fisher's exact test where appropriate. A $P$ value $<0.05$ was considered statistically significant. All statistical analyses were performed using STATISTICA version 12 (2014; Stat Soft Inc., Tulsa, OK) and Microsoft Excel (Microsoft Office 2008; Microsoft Corp., Redmond, WA) on Mac OS (Apple Inc., Cupertino, CA)

The study was conducted according to the principles of the Declaration of Helsinki and approved by the Regional Ethical Review Board in Stockholm (approval number 2016/744-31/4).

\section{RESULTS}

\section{ED Population}

Out of 3581 visits to the ED with a chief complaint of chest pain, hs-cTnT was analyzed in 3169. In 1973 patients, a second hscTnT was analyzed, and among these patients, the median (IQR) time between admission to the ED and the first sample of hs-cTnT was 26 (16-47) minutes, and the median (IQR) time between the first and the second sample was 73 (63-96) minutes. The majority $(70.8 \%)$ of all second hs-cTnT samples were obtained within the time limit of $>30$ minutes and $\leq 90$ minutes, resulting in 1397 visits by unique patients who fulfilled the inclusion but not exclusion criteria. The selection of the study group is presented in Figure 1. In patients who had a baseline value of hs-cTnT of $\leq 14 \mathrm{ng} / \mathrm{L}$, the median (IQR) time between admission to the ED and the first sample of hs-cTnT was 28 (16-47) minutes, and the median (IQR) time between the first and the second sample was 67 (60-75) minutes (Fig. 2). In this group, $70.4 \%$ of all second hs-cTnT samples were obtained within the time limit of $>30$ minutes and $\leq 90$ minutes, resulting in 1091 included patients with a baseline value of hs-cTnT of $\leq 14 \mathrm{ng} / \mathrm{L}$.

\section{Study Population}

Baseline characteristics of the 1397 included patients are shown in Table 1. Patients with a hs-cTnT baseline value of $\leq 14 \mathrm{ng} / \mathrm{L}$ were younger and showed significant healthier values in almost all variables (eg, systolic blood pressure, renal function, N-terminal prohormone of brain natriuretic peptide, and ECG). The incidence of MI in the study population $(\mathrm{n}=1397)$ was $4.2 \%$, compared with $4.4 \%$ in all screened patients who had an analysis of hs-cTnT $(n=3169)$.

\section{Dynamic Changes in hs-cTnT in Patients With a Nonelevated Baseline Value of hs-cTnT}

The results of $\Delta$ hs-cTnT in patients with a hs-cTnT baseline value of $\leq 14 \mathrm{ng} / \mathrm{L}(\mathrm{n}=1091)$ are presented in Figure 3. The median (IQR) value of the first as well as the second hs-cTnT values was $<5$ $(<5-7) \mathrm{ng} / \mathrm{L}$. A total of 23 patients $(2.1 \%)$ had a dynamic change $(\geq 3 \mathrm{ng} / \mathrm{L})$.

\section{Outcome and 30-Day Follow-Up in Patients With a Nonelevated Baseline Value of hs-cTnT}

In patients with a baseline hs-cTnT of $\leq 14 \mathrm{ng} / \mathrm{L}(\mathrm{n}=1091)$, comparisons were made between the dynamic and nondynamic groups (Table 2). Admission to the hospital was more common in the dynamic group compared with the nondynamic group $(65.2 \%$ and $13.9 \%$, respectively). A total of 6 admitted patients were eventually discharged with an MI diagnosis, out of which 5 were adjudicated as non-ST-segment-elevation MI type 1 and 1 as unstable angina pectoris (UAP; see Table, Supplemental Digital Content 1, http://links.lww.com/HPC/A205, which shows the characteristics of patients with a hs-cTnT baseline value of $\leq 14 \mathrm{ng} / \mathrm{L}$ and a final diagnosis of MI or UAP). This resulted in an MI diagnosis in 4 of the admitted patients $(26.7 \%)$ in the dynamic group, compared with 1 of the admitted patients $(0.7 \%)$ in the nondynamic group. The total incidence of $\mathrm{MI}$ in those presenting with a baseline value of $\leq 14 \mathrm{ng} / \mathrm{L}$ was $0.5 \%$. In addition, 9 patients $(6.1 \%)$ in the nondynamic group were diagnosed with UAP. Altogether, $14(8.6 \%)$ of the admitted patients were diagnosed with ACS (ie, MI or UAP; see Table, Supplemental Digital Content 1, http://links.lww.com/HPC/A205). Diagnoses for all admitted patients are shown in Table 2 .

Eight patients (34.8\%) in the dynamic group were discharged directly from the ED. These patients were not diagnosed with an $\mathrm{ACS}$, and none of them were readmitted or died during follow-up. Out of these 8 patients, 5 had a $\Delta$ hs-cTnT of $3 \mathrm{ng} / \mathrm{L}, 1$ had a $\Delta$ hscTnT of $4 \mathrm{ng} / \mathrm{L}$, and 2 a $\Delta$ hs-cTnT of $5 \mathrm{ng} / \mathrm{L}$. In the nondynamic group, 920 patients $(86.1 \%)$ were discharged directly from the ED, 


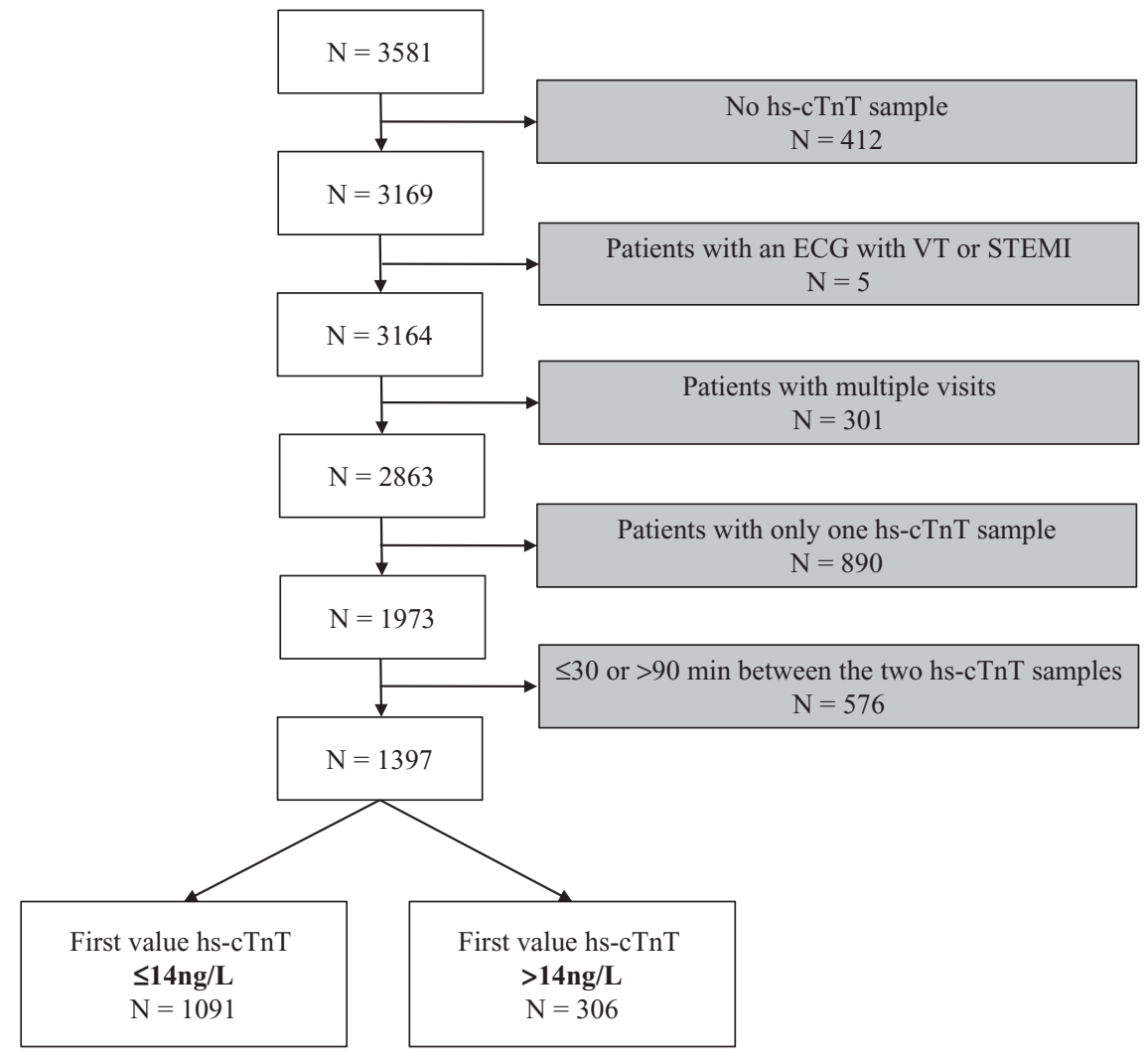

FIGURE 1. Selection of the study group of chest pain patients. $\mathrm{N}$ indicates total number of patients/visits; STEMI, ST-segmentelevation myocardial infarction; VT, ventricular tachycardia.

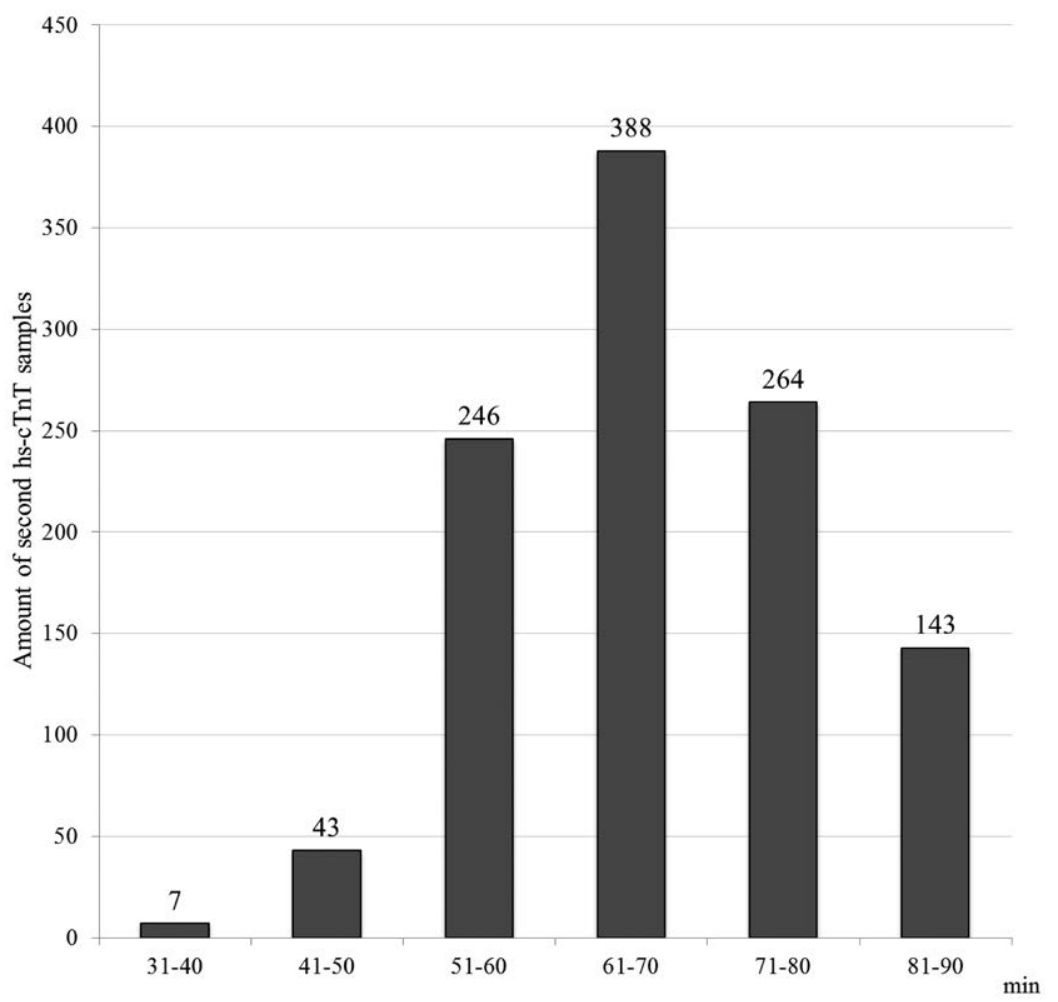

Time between first and second hs-cTnT sample
FIGURE 2. Time distribution between first and second sample of hs-cTnT in chest pain patients with a baseline value of $\leq 14 \mathrm{ng} / \mathrm{L}$ and $2 \mathrm{hs}-\mathrm{CTnT}$ values obtained within a time period of $>30$ to $\leq 90$ minutes, $\mathrm{N}=1091$. 


\begin{tabular}{|c|c|c|c|}
\hline Variable & $\leq 14 \mathrm{ng} / \mathrm{L}(\mathrm{N}=1091)$ & $>14 \mathrm{ng} / \mathrm{L}(\mathrm{N}=306)$ & $\boldsymbol{P}$ \\
\hline Age (years) & $52 \pm 16[1085]$ & $71.8 \pm 14.3$ & $<0.001$ \\
\hline Male gender & $604(55.4 \%)$ & $193(63.1 \%)$ & 0.04 \\
\hline Systolic BP (mm Hg) & $147 \pm 25[1081]$ & $153 \pm 29[298]$ & $<0.001$ \\
\hline Diastolic BP (mm Hg) & $82 \pm 12[1072]$ & $82 \pm 15[290]$ & 0.66 \\
\hline Heart rate $(\mathrm{bpm})$ & $77 \pm 17[1076]$ & $80 \pm 19[295]$ & 0.001 \\
\hline $\mathrm{eGFR}\left(\mathrm{mL} / \mathrm{min} / 1.73 \mathrm{~m}^{2}\right)$ & $93 \pm 23[1082]$ & $68 \pm 30[303]$ & $<0.001$ \\
\hline NT-proBNP (ng/L) & $105(39-222)[131]$ & $1020(413-3290)[108]$ & $<0.001$ \\
\hline Hemoglobin $(\mathrm{g} / \mathrm{L})$ & $142 \pm 15[1083]$ & $132 \pm 18[304]$ & $<0.001$ \\
\hline White blood cell count $\left(10^{9} / \mathrm{L}\right)$ & $7.7 \pm 2.5[1083]$ & $8.8 \pm 5.4[304]$ & $<0.001$ \\
\hline C-reactive protein $(\mathrm{mg} / \mathrm{L})$ & $1(<1-4)[1088]$ & $4(1-10.5)[303]$ & $<0.001$ \\
\hline \multicolumn{2}{|l|}{ ECG rhythm } & & $<0.001$ \\
\hline Sinus & $861(78.9 \%)$ & $182(59.5 \%)$ & \\
\hline Atrial flutter/atrial fibrillation & $29(2.7 \%)$ & $45(14.7 \%)$ & \\
\hline Other pathologies/rhythm not commented & $82(7.5 \%)$ & $47(15.4 \%)$ & \\
\hline No ECG available for review & $119(10.9 \%)$ & $32(10.5 \%)$ & \\
\hline \multicolumn{2}{|l|}{ ECG morphology } & & $<0.001$ \\
\hline Normal & $695(63.7 \%)$ & $99(32.4 \%)$ & \\
\hline LBBB/ventricular pacing & $22(2 \%)$ & $29(9.5 \%)$ & \\
\hline ST-segment depression* & $33(3 \%)$ & $41(13.4 \%)$ & \\
\hline T-wave inversion & $35(3.2 \%)$ & $23(7.5 \%)$ & \\
\hline Other pathologies $\dagger$ & $147(13.5 \%)$ & $69(22.5 \%)$ & \\
\hline Morphology not commented & $40(3.7 \%)$ & $13(4.2 \%)$ & \\
\hline No ECG available for review & $119(10.9 \%)$ & $32(10.5 \%)$ & \\
\hline Time of stay in the ED (min) & $240(194.5-310)$ & $270(201-382.5)$ & $<0.001$ \\
\hline Time until attended by physician (min) & $74(37-142)[1081]$ & $58(30.75-96.25)[304]$ & $<0.001$ \\
\hline $\begin{array}{l}\text { Includes all patients with a second hs-cTnT sa } \\
\text { range) or sum and percent of the total/[amount of } g \\
\text { *Significant or nonsignificant ST-segment depr } \\
\text { †Unspecific branch block, low voltage, Q wave } \\
\text { BP indicates blood pressure; eGFR, estimate } \\
\text { prohormone of brain natriuretic peptide. }\end{array}$ & $\begin{array}{l}\text { minutes, } N=1397 \text {. Data a } \\
\text { logies. } \\
\text { nges with or without other } 1 \\
\text { B, left bundle branch bloc }\end{array}$ & $\begin{array}{l}\text { an } \pm \text { standard deviation or } \mathrm{n} \\
\text { patients in the group; NT- }-1\end{array}$ & $\begin{array}{l}\text { terquartile } \\
\text { N-terminal }\end{array}$ \\
\hline
\end{tabular}

and 11 patients $(1.2 \%)$ were later readmitted, although no death or MI occurred within 30 days. The most common diagnosis at readmission was unspecified chest pain (R07.4).

The median (min-max range) HEART score values among patients discharged with a dynamic change and among those admitted and diagnosed with ACS without a dynamic change was $1(0-4)$ and 5 (range, 4-7) respectively. The HEART score of these subgroups are presented in Table 3.

In addition, we analyzed the 621 patients with a hs-cTnT baseline value of $<5 \mathrm{ng} / \mathrm{L}$ (ie, below the limit of detection). Among these, 6 patients $(1.0 \%)$ had a dynamic $\Delta$ hs-cTnT. Two patients $(33.3 \%)$ and 62 patients $(10.1 \%)$ were admitted in the dynamic and nondynamic group, respectively. None of the admitted patients were diagnosed with MI. One patient (1.6\%) in the nondynamic group, and none in the dynamic group, was diagnosed with UAP. Among those discharged directly from the ED, no death, MI, or readmission occurred in any of the groups within the 30-day follow-up. Thus, in total, only 1 of the $621(0.2 \%)$ patients with a hs-cTnT baseline value of $<5 \mathrm{ng} / \mathrm{L}$ was diagnosed with ACS.

\section{DISCUSSION}

In the current study, we evaluated the use of a 1-hour measurement of hs-cTnT adopted in the clinical routine in an unselected ED population of chest pain patients with a nonelevated hs-cTnT at presentation. The main finding was that dynamic 1-hour changes in hs-cTnT were uncommon but associated both with higher rate of admission and higher rate of MI. Further, no death or MI occurred within a 30-day follow-up of patients who were discharged directly from the ED. These findings support that the algorithm implemented in the clinical routine is useful and safe in the assessment of chest pain patients in the ED, a patient population with a traditionally high admission rate.

Dynamic changes occurred in $23(2.1 \%)$ of the patients in our study. The high proportion of patients with nondynamic changes might be explained by several factors. First, the base for inclusion was an unselected chest pain population at low risk that consisted of all patients registered with chief complaint of chest pain in the ED, independently of the suspected cause, onset and duration of the chest pain, and the patients' past medical history. Second, and even more important, in contrast to previous studies, this study excluded patients with a baseline hs-cTnT of $>14 \mathrm{ng} / \mathrm{L}$. This resulted in a higher proportion of patients in the nondynamic group compared with the rule-out groups in previous studies that included patients regardless of the baseline hs-cTnT value. . $^{2,8,15}$

There was a significant difference in admission rate between the dynamic and nondynamic group in our study (65.2\% vs, $13.9 \%)$, indicating that the algorithm was well implemented into clinical practice. We assume that the relatively low proportion of admitted 


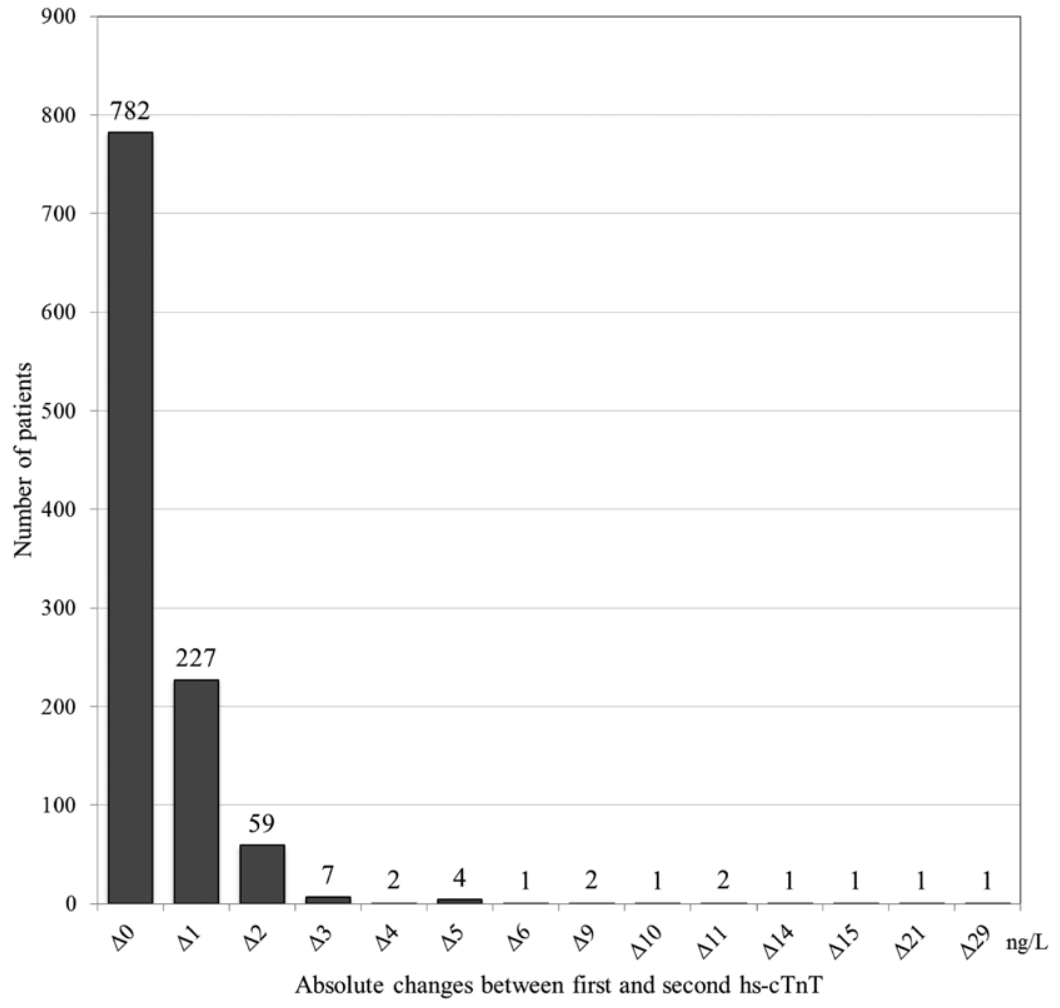

FIGURE 3. Distribution of absolute change in hsCTnT in chest pain patients with a baseline value of $\leq 14 \mathrm{ng} / \mathrm{L}$ and $2 \mathrm{hs}-\mathrm{cTnT}$ values obtained within a time period of $>30$ to $\leq 90$ minutes, $N=1091$. $\Delta$ indicates delta/absolute change. patients in the nondynamic group reflects clinical assessment with both the 1-hour hs-cTnT algorithm and the HEART score. We also found a significant difference in the rate of patients admitted due to MI between the dynamic and nondynamic groups (26.7\% vs. $0.7 \%)$, and $17.4 \%$ of all patients presenting with a dynamic $\Delta$ hs-cTnT received the diagnosis $\mathrm{MI}$, compared with $0.1 \%$ in patients with a nondynamic change. The very low proportion of MI in the nondynamic group was in accordance with prior findings. ${ }^{2,8}$ This indicates that the algorithm adds important information that can facilitate a safe rule out of MI in an ED chest pain population. Even though the total incidence of MI in those presenting with a baseline value of $\leq 14 \mathrm{ng} / \mathrm{L}$ was only $0.5 \%$, prior studies have shown that a single value of hs-cTnT within normal reference range is not enough to safely rule out an ongoing MI. ${ }^{16}$

The incidence of MI in our study was markedly lower compared with that in prior studies evaluating the original algorithm, ${ }^{2,7,8,15}$ both in the dynamic group when compared to the rule-in groups of previous studies and in all patients regardless of $\Delta \mathrm{hs}-\mathrm{cTnT}$. The difference in $\mathrm{MI}$ incidence between our dynamic group and the rule-in groups in the abovementioned studies might partly be explained by the fact that prior studies used an observational zone for patients between rule in and rule out, which hampers comparisons to the dynamic group in our study. The overall low incidence of MI is mainly explained by the fact that we only included patients with a baseline hs-cTnT of $\leq 14 \mathrm{ng} / \mathrm{L}$ in the final analysis, which reduced the MI incidence from $4.2 \%$ in the study population to $0.5 \%$ in the final analysis. It is also partly explained by the fact that we included more unselected chest pain patients with a lower risk. In a prior study that included an unselected chest pain population in the ED, the MI incidence within 30 days was only somewhat higher in all patients independently of the baseline result of hs-cTnT (5.4\% vs. $4.2 \%$ in our study). ${ }^{17}$

A total of $11(73.3 \%)$ out of 15 admitted patients with a $\Delta$ hscTnT of $\geq 3 \mathrm{ng} / \mathrm{L}$ were not diagnosed with an ACS. This reflects the fact that release of as well as dynamic changes in hs-cTnT might be due to other diagnoses such as tachyarrhythmia, decompensated heart failure, pulmonary embolism, or a severe infection. ${ }^{4,13,18}$ In addition to the one patient in the nondynamic group who was admitted and diagnosed with an MI, 9 of the patients in the nondynamic group were admitted and diagnosed with UAP. The median HEART score value for these 10 patients was high (5 points), suggesting that calculation of HEART score could identify those patients with an acute risk of a MACE. These findings indicate that we cannot completely rely on early dynamic changes in hs-cTnT for the diagnosis of ACS. History, risk factors, clinical findings, and ECG still need to be a part of the clinical assessment.

In the 8 patients discharged directly from the ED in the dynamic group, the median HEART score value was very low (1 point), and no death or MI occurred within 30 days of follow-up. This indicates that the HEART score may be used to safely discharge chest pain patients also in those with early dynamic changes. This is a novel finding, but we acknowledge the small size of this subgroup. Further, none of the $920(86.1 \%)$ patients in the nondynamic group who were discharged directly from the ED died or had an MI within 30 days of follow-up.

Whether a further measurement of troponin after 1 hour is necessary in patients with undetectable levels of hs-cTnT at baseline remains a matter of debate. Previous studies have shown an excellent prognosis in these patients, ${ }^{17,19-21}$ which was verified also in the present study. The results suggest that undetectable levels of hs-cTnT at baseline might be included in the rule-out algorithm, as indicated in a previous study using a novel hs-cTn I assay. ${ }^{22}$

The present study has several limitations. This was a single-center study, which may influence the generalizability of the results. Further, this was a retrospective study in an unselected ED population of chest pain patients. However, this might reflect the everyday clinical situation in a different way than prior controlled studies, and the study setting could provide results that are more representative of the 1 -hour algorithm when adopted into clinical 


\begin{tabular}{|c|c|c|c|}
\hline Variable & $\Delta<3(N=1068)$ & $\Delta \geq 3(N=23)$ & $\boldsymbol{P}$ \\
\hline Age (years) & $51.8 \pm 16[1062]$ & $59.8 \pm 14.2$ & 0.018 \\
\hline Male gender & $594(55.6 \%)$ & $10(43.5 \%)$ & 0.240 \\
\hline Systolic BP (mm Hg) & $147 \pm 25[1059]$ & $148 \pm 27[22]$ & 0.787 \\
\hline Diastolic BP (mm Hg) & $82 \pm 12[1050]$ & $86 \pm 17[22]$ & 0.091 \\
\hline Heart rate (bpm) & $77 \pm 16[1054]$ & $91 \pm 27[22]$ & $<0.001$ \\
\hline eGFR $\left(\mathrm{mL} / \mathrm{min} / 1.73 \mathrm{~m}^{2}\right)$ & $93 \pm 23[1059]$ & $85 \pm 19$ & 0.095 \\
\hline NT-proBNP (ng/L) & $105(38-221)[123]$ & $147(60-968)[8]$ & 0.436 \\
\hline Time in the ED (min) & $240(194.5-309)$ & $267(182-355)$ & 0.487 \\
\hline Time until attended by physician (min) & $75(38-143)[1058]$ & $30(21-71)$ & 0.001 \\
\hline Admitted & $148(13.9 \%)$ & $15(65.2 \%)$ & $<0.001$ \\
\hline Myocardial infarction (ICD I21-I22) & $1(0.7 \%)$ & $4(26.7 \%)$ & $<0.001$ \\
\hline Unstable angina pectoris (ICD I20.0) & $9(6.1 \%)$ & 0 & 1.000 \\
\hline Angina pectoris (ICD I20.8-9) & $12(8.1 \%)$ & $1(6.7 \%)$ & 1.000 \\
\hline Atrial fibrillation (ICD I48) & $8(5.4 \%)$ & $1(6.7 \%)$ & 0.590 \\
\hline Supraventricular tachycardia (ICD I47.1) & $2(1.4 \%)$ & $1(6.7 \%)$ & 0.252 \\
\hline Unspecified chest pain (ICD R07.4) & $70(47.3 \%)$ & $6(40 \%)$ & 1.000 \\
\hline Other diagnoses & $46(31.1 \%)$ & $2(13.3 \%)$ & 0.234 \\
\hline Death within 30 days & $1(0.7 \%)$ & 0 & NS \\
\hline Readmission within 30 days due to myocardial infarction, unstable angina pectoris, or angina pectoris & 0 & 0 & NS \\
\hline Readmission within 30 days due to any diagnosis & $7(4.7 \%)$ & 0 & 0.502 \\
\hline Discharged directly from the ED & $920(86.1 \%)$ & $8(34.8 \%)$ & $<0.001$ \\
\hline Death or readmission within 30 days due to myocardial infarction or unstable angina pectoris & 0 & 0 & NS \\
\hline Readmission within 30 days due to angina pectoris & $1(0.1 \%)$ & 0 & NS \\
\hline Readmission within 30 days due to any diagnosis & $11(1.2 \%)$ & 0 & 0.756 \\
\hline
\end{tabular}

$\mathrm{N}=1091$. Data are presented as mean \pm standard deviation or median (interquartile range) or sum and percent of the total/[amount of group].

$\Delta$ indicates delta; BP, blood pressure; bpm, beats per minute; eGFR, estimated glomerular filtration rate; ICD, international classification of diseases; N, total number of patients in the group; NS, not significant; NT-proBNP, N-terminal prohormone of brain natriuretic peptide.

TABLE 3. HEART Score of Subgroups of Chest Pain Patients With a Baseline Value of $\leq 14 \mathrm{ng} / \mathrm{L}$

\begin{tabular}{lccc}
\hline & $\begin{array}{c}\text { Discharged With Dynamic } \\
\text { Change }(\mathbf{n}=\mathbf{8})\end{array}$ & $\begin{array}{c}\text { Admitted Without Dynamic Change and ACS } \\
\text { Diagnosis }(\mathbf{n}=\mathbf{1 0})\end{array}$ & $\begin{array}{c}\text { Admitted With Dynamic } \\
\text { Change (n= 15) }\end{array}$ \\
\hline HEART score & $1(0-4)$ & $5(4-7)$ & $4(1-6)$ \\
H & $0(0-0)$ & $1(0-2)$ & $0(0-2)$ \\
E* & $0(0-1)$ & $0(0-1)$ & $0(0-2)$ \\
A & $1(0-2)$ & $1.5(1-2)$ & $1(1-2)$ \\
R & $0(0-2)$ & $2(1-2)$ & $1(0-2)$ \\
T & 0 & 0 & 0 \\
\hline
\end{tabular}

A second value was obtained within a time period of $>30$ to $\leq 90$ minutes. Data are presented as median (minimal and maximal range).

$\mathrm{N}$ indicates total number of patients in the group; $\mathrm{H}$, history; E, electrocardiogram; A, age; R, risk factors; T, initial Troponin.

* No missing electrocardiogram interpretation.

practice, which could also be considered as a strength of the study. A relatively high proportion of the screened patients had only 1 hs-cTnT sample drawn in the ED. It is possible that this was a clinical decision in accordance with the present guidelines (eg, long duration since pain onset, undetectable baseline hs-cTnT, admission without further assessment). It is also possible that this has led to that some patients with dynamic changes have been missed. However, the overall low risk profile of our study population indicates that a wide, rather than narrow indication for a second hs-cTnT was used, and the MI incidence in those with a second troponin obtained within $>30$ minutes but $\leq 90$ minutes was comparable to that in all screened patients who had an analysis of hs-cTnT. A time period between the first and second sample of hs-cTnT of $>30$ minutes but $\leq 90$ minutes was accepted because an exact time is very difficult to obtain in clinical practice. The majority of the second samples of hs-cTnT were obtained $>60$ minutes, but despite an interval of $\leq 60$ minutes in some patients, no MACE occurred in patients discharged from the ED. The total time for transport, analysis, and response to the initial hs-cTnT usually exceeded 1 hour; thus, in a large proportion of cases, the result of the baseline value was not known when the second sample was drawn. Although the HEART score was implemented 
in the clinical routine, it was unfortunately not systematically documented and, therefore, only calculated retrospectively and in subgroups. Follow-up was limited to 30 days because this is a common follow-up time in similar studies. ${ }^{7,8,15,19,22}$ The incidence of dynamic hs-cTnT changes as well as the event rate of MI was low, and a larger population would be necessary to evaluate the rule in part of the algorithm. However, our findings suggest that the ruleout strategy is safe in a population that was earlier often admitted for further analysis of hs-cTnT.

\section{CONCLUSIONS}

We found that dynamic 1-hour changes in hs-cTnT were uncommon in the clinical routine but associated with a higher rate of $\mathrm{MI}$ in an unselected ED population of chest pain patients with a nonelevated hs-cTnT at presentation. No death or MI occurred during a 30-day follow-up among patients discharged directly from the ED. Thus, a 1-hour measurement may facilitate an early rule out of MI in chest pain patients in the ED, a patient population with a traditionally high admission rate. A careful clinical assessment is still necessary, for example, with the HEART score, to identify patients with UAP, as well as those few MI patients where dynamic 1-hour changes in hs-cTnT cannot be seen. Further studies examining the effect of introducing the algorithm in a prospective setting with a more selected chest pain population are needed.

\section{ACKNOWLEDGMENT}

We thank Marie-Christine Berglund at Planering och Uppö̈ljning, Karolinska University Hospital, for data extraction from the KARDA.

\section{REFERENCES}

1. Goodacre S, Cross E, Arnold J, et al. The health care burden of acute chest pain. Heart. 2005;91:229-230.

2. Reichlin T, Twerenbold R, Wildi K, et al. Prospective validation of a 1-hour algorithm to rule-out and rule-in acute myocardial infarction using a highsensitivity cardiac troponin T assay. CMAJ. 2015;187:E243-E252.

3. Omstedt $\AA$, Höijer J, Djärv T, et al. Hypertension predicts major adverse cardiac events after discharge from the emergency department with unspecified chest pain. Eur Heart J Acute Cardiovasc Care. 2016;5:441-448.

4. Thygesen K, Mair J, Giannitsis E, et al.; Study Group on Biomarkers in Cardiology of ESC Working Group on Acute Cardiac Care. How to use high-sensitivity cardiac troponins in acute cardiac care. Eur Heart J. 2012;33:2252-2257.

5. Bassand JP, Hamm CW, Ardissino D, et al. Guidelines for the diagnosis and treatment of non-ST-segment elevation acute coronary syndromes. Eur Heart J. 2007;28:1598-660.

6. Hamm CW, Bassand JP, Agewall S, et al.; ESC Committee for Practice Guidelines. ESC Guidelines for the management of acute coronary syndromes in patients presenting without persistent ST-segment elevation: The Task Force for the management of acute coronary syndromes (ACS) in patients presenting without persistent ST-segment elevation of the European Society of Cardiology (ESC). Eur Heart J. 2011;32:2999-3054.

7. Reichlin T, Schindler C, Drexler B, et al. One-hour rule-out and rule-in of acute myocardial infarction using high-sensitivity cardiac troponin T. Arch Intern Med. 2012;172:1211-1218.

8. Mueller C, Giannitsis E, Christ M, et al.; TRAPID-AMI Investigators Multicenter evaluation of a 0-hour/1-hour algorithm in the diagnosis of myocardial infarction with high-sensitivity cardiac troponin T. Ann Emerg Med. 2016;68:76-87.e4.

9. Roffi M, Patrono C, Collet JP, et al.; Management of Acute Coronary Syndromes in Patients Presenting without Persistent ST-Segment Elevation of the European Society of Cardiology. 2015 ESC Guidelines for the management of acute coronary syndromes in patients presenting without persistent ST-segment elevation: Task Force for the Management of Acute Coronary Syndromes in Patients Presenting without Persistent ST-Segment Elevation of the European Society of Cardiology (ESC). Eur Heart J. 2016;37:267-315.

10. Backus BE, Six AJ, Kelder JC, et al. A prospective validation of the HEART score for chest pain patients at the emergency department. Int $J$ Cardiol. 2013;168:2153-2158.

11. Backus BE, Six AJ, Kelder JC, et al. Chest pain in the emergency room: a multicenter validation of the HEART Score. Crit Pathw Cardiol. 2010;9:164-169.

12. Melki D, Jernberg T. HEART score: a simple and useful tool that may lower the proportion of chest pain patients who are admitted. Crit Pathw Cardiol. 2013;12:127-131.

13. Giannitsis E, Kurz K, Hallermayer K, Jarausch J, Jaffe AS, Katus HA Analytical validation of a high-sensitivity cardiac troponin $\mathrm{T}$ assay. Clin Chem. 2010;56:254-261.

14. Levey AS, Coresh J, Greene T, et al.; Chronic Kidney Disease Epidemiology Collaboration. Using standardized serum creatinine values in the modification of diet in renal disease study equation for estimating glomerular filtration rate. Ann Intern Med. 2006;145:247-254.

15. Mokhtari A, Borna C, Gilje P, et al. A 1-h combination algorithm allows fast rule-out and rule-in of major adverse cardiac events. J Am Coll Cardiol. 2016;67:1531-1540.

16. Zhelev Z, Hyde C, Youngman E, et al. Diagnostic accuracy of single baseline measurement of Elecsys Troponin T high-sensitive assay for diagnosis of acute myocardial infarction in emergency department: systematic review and meta-analysis. BMJ. 2015;350:h15

17. Bandstein N, Ljung R, Johansson M, et al. Undetectable high-sensitivity cardiac troponin $T$ level in the emergency department and risk of myocardial infarction. J Am Coll Cardiol. 2014;63:2569-2578.

18. Sandoval Y, Smith SW, Thordsen SE, et al. Supply/demand type 2 myocardial infarction: should we be paying more attention? J Am Coll Cardiol. 2014;63:2079-2087.

19. Body R, Mueller C, Giannitsis E, et al.; TRAPID-AMI Investigators. The use of very low concentrations of high-sensitivity troponin t to rule out acute myocardial infarction using a single blood test. Acad Emerg Med. 2016;23:1004-1013.

20. Rubini Giménez M, Hoeller R, Reichlin T, et al. Rapid rule out of acute myocardial infarction using undetectable levels of high-sensitivity cardiac troponin. Int J Cardiol. 2013;168:3896-3901.

21. Thelin J, Melander O, Öhlin B. Early rule-out of acute coronary syndrome using undetectable levels of high sensitivity troponin T. Eur Heart J Acute Cardiovasc Care. 2015;4:403-409.

22. Lindahl B, Jernberg T, Badertscher $\mathrm{P}$, et al. An algorithm for rule-in and rule-out of acute myocardial infarction using a novel troponin I assay. Heart. 2017; 103:125-131. 